\title{
Aaper Advanced Inversion Techniques for Ground Penetrating Radar
}

\author{
Alessandro Fedeli, Matteo Pastorino, and Andrea Randazzo \\ Department of Electrical, Electronic, Telecommunications Engineering, and Naval Architecture (DITEN), \\ University of Genoa, Genova, Italy
}

https://doi.org/10.26636/jtit.2017.119717

\begin{abstract}
Ground Penetrating Radar (GPR) systems are nowadays standard inspection tools in several application areas, such as subsurface prospecting, civil engineering and cultural heritage monitoring. Usually, the raw output of GPR is provided as a B-scan, which has to be further processed in order to extract the needed information about the inspected scene. In this framework, inverse-scattering-based approaches are gaining an ever-increasing interest, thanks to their capabilities of directly providing images of the physical and dielectric properties of the investigated areas. In this paper, some advances in the development of such inversion techniques in the GPR field are revised and discussed.
\end{abstract}

Keywords-electromagnetic scattering, Ground Penetrating Radar, inverse problems.

\section{Introduction}

Ground Penetrating Radar (GPR) imaging has attracted a lot of interest in the last years. Nowadays, GPR systems are used in a great variety of research and application areas, including civil engineering [1]-[3], archaeological and geophysical prospecting [4], and cultural heritage monitoring [5]. Besides these classical areas, novel applications, such as through-wall imaging [6], contaminant detection [7], [8], tunnel and underground facility detection [9], and planet exploration [10], are attracting an ever-increasing attention.

In order to obtain accurate GPR images, it is necessary to carefully design the different parts of the system, ranging from the acquisition hardware to the signal processing algorithms needed to interpret the data. Moreover, there are usually several requirements, such as portability of the imaging systems, ultra-wide-band behavior, and limited coupling between transmitting and receiving antennas, which must be suitably considered in the GPR design and realization.

Concerning the data interpretation, there is also the need for performing some preparatory steps. In fact, usually scattered field extraction procedures must be employed in order to isolate the scattering contribution in the measured electromagnetic field data and to suppress the effects of clutter [11]. Moreover, it is needed to obtain a satisfactory estimate of the dielectric properties of the background medium [12] and to develop appropriate forward scattering models [13], [14]. Antennas also represent key elements in the development of effective imaging systems. In fact, since they are located in close proximity with the ground (or more generally, with the host medium) accurate antenna characterizations are needed [15], [16].

Although GPR is now a standard inspection tool, there are still open issues that must be faced in order to further enhance the imaging capabilities. In particular, GPRs usually provide the output images in the form of B-scans, i.e. two-dimensional representations of the amplitude of the acquired scattered field data versus position and time. Such images can be quite difficult to interpret, especially when dealing with complex embedding media. Significant improvements can be obtained by reformulating the image formation process as an electromagnetic inverse scattering problem, where the main parameters describing the inspected scene are retrieved by inverting a proper model describing the scattering phenomena.

Several research groups around the world follow this point of view and very interesting numerical and experimental results have been already reported in the scientific literature. In this paper, some recent advances concerning the development of inverse scattering procedures for GPR imaging are revised and discussed.

\section{GPR Imaging as an Inverse Problem}

Subsurface imaging requires to solve an electromagnetic inverse scattering problem [17], i.e. starting from measurements of the scattered electric field collected in a proper measurement domain (e.g. a line over the air-ground interface), the aim is to retrieve some parameters describing the buried targets. Such parameters can be the full distributions of the dielectric properties or some features able to describe the target (e.g. its shape and position). As it is well known, such kind of problems turns out to be non-linear [18] and ill-posed [19]. Consequently, special care is required in the development of effective solving strategies.

\subsection{Quantitative Electromagnetic Inversion}

In the scientific literature, several methods have been proposed for tackling this task, not only in the field of subsurface imaging [20]-[24]. When a quantitative reconstruc- 
tion is needed, e.g. when the aim is to retrieve the whole dielectric distribution of the inspected region, the full nonlinear problem must be taken into account. In this case, the imaging problem is usually recast as an optimization problem. Newton-like iterative methods [25]-[27] and gradient based solution procedures [28]-[30] are often used in this case. However, such approaches suffer from local minima problems [31], and thus are quite sensitive to the availability of a suitable starting guess. Stochastic minimization schemes have also been largely used [32]-[34]. These methods are in principle able to overcome the problem of local minima, but their numerical complexity is often higher than the one of deterministic approaches.

The imaging problem can be simplified by using linearized scattering models, e.g. by exploiting Born or Rytov approximations [17] in the case of penetrable scatterers and the Kirchhoff one for metallic objects. It is worth noting that the class of scatterers for which linear models effectively work is limited, and consequently, they usually do not allow a quantitative reconstruction. Anyway, such approaches are able to detect, localize and provide rough information about the scatterers' shape [35]. Moreover, linear estimations can be used to provide a starting point for non-linear quantitative approaches. In all cases, ill-posedness still remains an issue and consequently there is a need to employ some regularization scheme able to increase the stability versus noise [19].

\subsection{Other GPR Imaging Techniques}

In the framework of GPR imaging, migration algorithms are still being used for obtaining qualitative reconstructions of the inspected scene. Although such approaches have been initially developed in the framework of seismic imaging starting from qualitative concepts, they can be derived from approximated linear scattering models [36]. Some of the main approaches belonging to such class are backpropagation [37], time-reversal [38], and omega-kappa algorithms [39].

Beside the previous approaches, other qualitative methods, aimed at directly retrieving only a limited set of information about the embedded scatterers (e.g. their positions and external supports), have also been proposed. The linear sampling [40], the factorization, and the MUltiple SIgnal Classification (MUSIC) methods [41] belong to this class of inversion algorithms.

\section{Recent Advancements in the Development of Inversion Techniques for GPR Imaging}

In the last few years, several research activities have been performed in order to increase the imaging capabilities of GPR systems. In particular, beside the development of novel inverse-scattering schemes, also methods for background removal and clutter rejection, soil models and medium estimation procedures, and GPR antenna modeling as well as deconvolution techniques have been proposed. Moreover, efficient approaches for solving the forward scattering problem by buried structures have also been developed.

\subsection{Nonlinear Inverse Scattering Methods}

Concerning the development of novel inverse-scattering schemes, several different approaches that extend and combine various methods previously mentioned have been discussed and evaluated in the scientific literature. Iterative multi-scale strategies have been proposed in [42]-[45], allowing an efficient usage of the limited amount of available information. In such techniques, the inspected area is iteratively reconstructed at different scales and at each scale specific inversion methods are used to obtain a quantitative reconstruction of the dielectric properties. Compressive sensing techniques have also been successfully applied in the field of GPR imaging [46]-[48]. In such approaches, the imaging problem is recast as a minimization in $L^{1}$ functional spaces. Consequently, sparse solutions (with respect to a properly selected basis) are obtained. Non-linear inversion algorithms performing a regularization in $L^{p}$ Banach spaces (with $p$ greater than 1 ) have also been recently proposed in [49], [50]. Such methods have been found to be very effective in reconstructing small targets and in reducing over smoothing and background artifacts with respect to standard inversion in the Hilbert space $(p=2)$.

\subsection{Linear Inverse Scattering Techniques}

Linear strategies have been considered, too. For example, in [51] a tomographic approach based on a truncated singular value decomposition (TSVD) inversion procedure has been compared to common procedures creating threedimensional images by interpolating two-dimensional reconstructions. The problem of imaging buried targets from airborne gathered scattered field data has also been addressed in in [52] by using linear techniques.

\subsection{Qualitative Approaches and Sampling Methods}

Concerning the use of qualitative techniques, subspacebased approaches [53] and sampling methods [54] have been found to be quite effective. It is worth noting that sampling techniques able to provide quantitative reconstructions have also been recently developed [55], [56]. Approaches devoted to the localization and shaping of targets have also been reported. As an example, a technique belonging to this class has been presented in [57]-[59]. The positions of buried targets are detected by estimating the scattered field directions of arrival through subarrays processing followed by a statistical filtering and a triangularization technique. 
Real-time detection of multiple buried scatterers has also been attained by using learning based techniques such as Support Vector Machines (SVM) [60].

\subsection{Preprocessing Methods}

Several novel algorithmic solutions have also been proposed for the preprocessing stage needed to successfully apply inverse scattering methods. Concerning the background removal and surface clutter mitigation problem, several strategies have been proposed in the past. Although average trace subtraction is often used for its simplicity, more effective approaches could enhance the reconstruction quality. As an example, in [61] a time-gating entropy based method is presented, which is able to provide better reconstructions than standard methods.

\subsection{Soil Properties Estimation}

The development of soil models and medium estimation procedures is also of great interest. In fact, such information is always required for correctly defining the electromagnetic models used in the inversion procedures. For example, in [62] an efficient algorithm for the computation of the time domain reflection coefficient in the transverse magnetic (TM) case has been developed in order to better characterize the surface reflections. In [63], [64] subspace methods and learning-based strategies have been used for the estimation of time delays, permittivity and roughness parameters within pavement structures. Estimation of specific soil parameters (e.g., moisture and clay content) have also been addressed in [65]. Moreover, in [12], [66], [67] inversion approaches have been employed for determining ground water contents in hydrological applications.

\subsection{Inclusion of Advanced Antenna Models}

Because of the complexity of the inverse problem which has to be solved in GPR imaging, reconstruction procedures can be significantly improved including detailed models of the involved antennas [68], [69]. These models can in principle allow to avoid the introduction of strong simplifying approximations, which clearly degrade the inversion results. The combination of far-field antenna models with a tomographic linear inverse scattering method (under the Born approximation) has been proposed in [70]. More recently, near-field models have been developed and included in inversion techniques [16], [71], [72].

\subsection{Validation of GPR Inversion Approaches}

In the development of inverse scattering methods, the validation of results is crucial. Frequently, inversion techniques are tested using synthetic data, and sometimes with experimental measurements (where experimental facilities are available). However, it is difficult to compare dif- ferent approaches without a common benchmark. In this respect, it is worth noting that in the framework of the COST Action TU1208 "Civil Engineering Applications of Ground Penetrating Radar" an open database of radargrams is available [73]. This database, which contains both synthetic and experimental data (e.g., radargrams of concrete cells, roads, trees, columns, bridges, and so on) can be exploited by researchers for testing and comparing the performance of GPR inversion techniques. A contribution to this initiative has also been represented by the GPR imaging challenge organized within the IWAGPR 2017 conference [74], where a simulated three-dimensional realistic landmine detection environment has been proposed.

\section{Conclusions}

In this paper, a brief overview about advanced inversion techniques for GPR has been presented. GPR imaging can be seen as an inverse scattering problem, in which the dielectric properties of the buried targets have to be estimated starting from measurements of the electromagnetic field. With respect to free space configurations, the problem is more challenging. Therefore, the estimation of the soil properties and the use of advanced antenna models act important roles. Of course, several kinds of solution techniques can be adopted. In particular, both quantitative (linear and nonlinear) and qualitative approaches have been considered, discussing some of the recent research trends.

\section{Acknowledgements}

This work benefited from the networking activities within the EU funded COST Action TU1208, "Civil Engineering Applications of Ground Penetrating Radar".

\section{References}

[1] A. Benedetto and L. Pajewski, Civil engineering applications of ground penetrating radar. Springer, 2015.

[2] J. Hugenschmidt and A. Kalogeropoulos, "The inspection of retaining walls using GPR", J. of Appl. Geophys., vol. 67, no. 4, pp. 335-344, 2009.

[3] A. Randazzo and C. Estatico, "A regularisation scheme for electromagnetic inverse problems: application to crack detection in civil structures", Nondestruc. Testing and Evaluation, vol. 27, no. 3, pp. 189-197, 2012.

[4] L. B. Conyers, Ground-penetrating radar for archaeology. Rowman Altamira, 2004.

[5] N. Masini, R. Persico, and E. Rizzo, "Some examples of GPR prospecting for monitoring of the monumental heritage", J. of Geophys. and Engin., vol. 7, no. 2, p. 190-199, 2010.

[6] E. J. Baranoski, "Through-wall imaging: Historical perspective and future directions", J. of the Franklin Institute, vol. 345, no. 6 , pp. 556-569, 2008.

[7] M. J. Bevan, A. L. Endres, D. L. Rudolph, and G. Parkin, "The non-invasive characterization of pumping-induced dewatering using ground penetrating radar", J. of Hydrol., vol. 281, no. 1-2, pp. 55-69, 2003. 
[8] J. L. Porsani, W. M. Filho, V. R. Elis, F. Shimeles, J. C. Dourado, and H. P. Moura, "The use of GPR and VES in delineating a contamination plume in a landfill site: a case study in SE Brazil", J. of Appl. Geophys., vol. 55, no. 3-4, pp. 199-209, 2004.

[9] L. Lo Monte, D. Erricolo, F. Soldovieri, and M. C. Wicks, "Radio frequency tomography for tunnel detection", IEEE Trans. on Geosci. and Remote Sensing, vol. 48, no. 3, pp. 1128-1137, 2010.

[10] T. R. Watters et al., "MARSIS radar sounder evidence of buried basins in the northern lowlands of Mars", Nature, vol. 444, no. 7121, pp. 905-908, 2006.

[11] O. Lopera, N. Milisavljević, and S. Lambot, "Clutter reduction in GPR measurements for detecting shallow buried landmines: a Colombian case study", Near Surface Geophys., vol. 5, no. 9, pp. 57-64, 2007.

[12] J. Minet, S. Lambot, E. C. Slob, and M. Vanclooster, "Soil surfaces water content estimation by full-waveform GPR signal inversion in the presence of thin layers", IEEE Trans. on Geosci. and Remote Sensing, vol. 48, no. 3, pp. 1138-1150, 2010.

[13] F. Frezza, L. Pajewski, C. Ponti, G. Schettini, and N. Tedeschi, "Cylindrical-wave approach for electromagnetic scattering by subsurface metallic targets in a lossy medium", J. of Appl. Geophys., vol. 97, pp. 55-59, 2013.

[14] I. Giannakis, A. Giannopoulos, and C. Warren, "A realistic FDTD numerical modeling framework of ground penetrating radar for landmine detection", IEEE J. of Selec. Topics in Appl. Earth Observ. and Remote Sensing, vol. 9, no. 1, pp. 37-51, 2016 (doi: 10.1109/JSTARS.2015.2468597).

[15] N. Diamanti and A. P. Annan, "Characterizing the energy distribution around GPR antennas", J. of Appl. Geophys., vol. 99, pp. 83-90, 2013.

[16] A. Tran, C. Warren, F. André, A. Giannopoulos, and S. Lambot, "Numerical evaluation of a full-wave antenna model for near-field applications", Near Surface Geophys., vol. 11, no. 2, pp. 155-165, 2013.

[17] M. Pastorino, Microwave Imaging. Hoboken: Wiley, 2010

[18] W. C. Chew, Waves and Fields in Inhomogenous Media. Wiley, 1999.

[19] M. Bertero and P. Boccacci, Introduction to inverse problems in imaging. Bristol, UK: IOP Publishing, 1998.

[20] C. Dourthe, C. Pichot, J. Y. Dauvignac, and J. Cariou, "Inversion algorithm and measurement system for microwave tomography of buried object", Radio Science, vol. 35, no. 5, pp. 1097-1108, 2000.

[21] M. Benedetti, M. Donelli, A. Martini, M. Pastorino, A. Rosani, and A. Massa, "An innovative microwave-imaging technique for nondestructive evaluation: Applications to civil structures monitoring and biological bodies inspection", IEEE Trans. on Instrument. and Measur., vol. 55, no. 6, pp. 1878-1884, 2006.

[22] M. A. Ali and M. Moghaddam, "3D nonlinear super-resolution microwave inversion technique using time-domain data", IEEE Trans. on Antenn. and Propag., vol. 58, no. 7, pp. 2327-2336, 2010.

[23] G. A. Meles, J. Van der Kruk, S. A. Greenhalgh, J. R. Ernst, H. Maurer, and A. G. Green, "A new vector waveform inversion algorithm for simultaneous updating of conductivity and permittivity parameters from combination crosshole/borehole-to-surface GPR data", IEEE Trans. on Geosc. and Remote Sensing, vol. 48, no. 9, pp. 3391-3407, 2010.

[24] C. Patriarca, S. Lambot, M. R. Mahmoudzadeh, J. Minet, and E. Slob, "Reconstruction of sub-wavelength fractures and physical properties of masonry media using full-waveform inversion of proximal penetrating radar", J. of Appl. Geophys., vol. 74, no. 1, pp. 26-37, 2011.

[25] T. J. Cui, W. C. Chew, A. A. Aydiner, and S. Chen, "Inverse scattering of two-dimensional dielectric objects buried in a lossy earth using the distorted Born iterative method", IEEE Trans. on Geosci. and Remote Sensing, vol. 39, no. 2, pp. 339-346, 2001.

[26] C. Estatico, M. Pastorino, and A. Randazzo, "A novel microwave imaging approach based on regularization in Lp Banach spaces", IEEE Trans. on Antenn. and Propag., vol. 60, no. 7, pp. 3373-3381, 2012 .
[27] C. Estatico, M. Pastorino, and A. Randazzo, "An inexact-Newton method for short-range microwave imaging within the second-order Born approximation", IEEE Trans. on Geosci. and Remote Sensing, vol. 43, no. 11, pp. 2593-2605, 2005.

[28] H. Harada, D. J. N. Wall, T. Takenaka, and M. Tanaka, "Conjugate gradient method applied to inverse scattering problem", IEEE Trans. on Antenn. and Propag., vol. 43, no. 8, pp. 784-792, 1995.

[29] T. Isernia, V. Pascazio, and R. Pierri, "A nonlinear estimation method in tomographic imaging", IEEE Trans. on Geosci. and Remote Sensing, vol. 35, no. 4, pp. 910-923, 1997.

[30] P. M. van den Berg and A. Abubakar, "Contrast source inversion method: state of art", Progress in Electromag. Res., vol. 34, pp. 189-218, 2001.

[31] T. Isernia, V. Pascazio, and R. Pierri, "On the local minima in a tomographic imaging technique", IEEE Trans. on Geosci. and Remote Sensing, vol. 39, no. 7, pp. 1596-1607, 2001.

[32] Y. Rahmat-Samii and E. Michielssen, Eds., Electromagnetic Optimization by Genetic Algorithms, 1st ed. New York, NY: Wiley, 1999.

[33] M. Pastorino, "Stochastic optimization methods applied to microwave imaging: a review", IEEE Trans. on Antenn. and Propag., vol. 55, no. 3, pp. 538-548, 2007.

[34] A. Randazzo, "Swarm optimization methods in microwave imaging", International Journal of Microwave Science and Technology, vol. 2012, Article ID 491713, 2012.

[35] M. Slaney, A. C. Kak, and L. E. Larsen, "Limitations of imaging with first-order diffraction tomography", IEEE Trans. on Microw. Theory and Techniq., vol. 32, no. 8, pp. 860-874, 1984.

[36] F. Soldovieri and R. Solimene, "Ground penetrating radar subsurface imaging of buried objects", in Radar Technology, G. Kouemou, Ed. InTech, 2010

[37] T. B. Hansen and P. M. Johansen, "Inversion scheme for ground penetrating radar that takes into account the planar air-soil interface", IEEE Trans. on Geosci. and Remote Sensing, vol. 38, no. 1, pp. 496-506, 2000.

[38] M. Fink, "Time reversal of ultrasonic fields. I. Basic principles", IEEE Trans. on Ultrasonics, Ferroelec., and Frequen. Control, vol. 39, no. 5, pp. 555-566, 1992.

[39] C. Cafforio, C. Prati, and F. Rocca, "SAR data focusing using seismic migration techniques", IEEE Trans. on Aerospace and Elec. Syst., vol. 27, no. 2, pp. 194-207, 1991.

[40] D. Colton, K. Giebermann, and P. Monk, "A regularized sampling method for solving three-dimensional inverse scattering problems", SIAM J. on Scient. Comput., vol. 21, no. 6, pp. 2316-2330, 2000 .

[41] E. A. Marengo, F. K. Gruber, and F. Simonetti, "Time-reversal MUSIC imaging of extended targets", IEEE Trans. on Image Process., vol. 16, no. 8, pp. 1967-1984, 2007.

[42] M. Salucci, L. Poli, N. Anselmi, and A. Massa, "Multifrequency particle swarm optimization for enhanced multiresolution GPR microwave imaging", IEEE Trans. on Geosci. and Remote Sensing, vol. 55, no. 3, pp. 1305-1317, 2017.

[43] M. Salucci, L. Poli, and A. Massa, "Advanced multi-frequency GPR data processing for non-linear deterministic imaging", Signal Process., vol. 132, pp. 306-318, 2017.

[44] M. Salucci, G. Oliveri, and A. Massa, "GPR prospecting through an inverse-scattering frequency-hopping multifocusing approach", IEEE Trans. on Geosci. and Remote Sensing, vol. 53, no. 12, pp. 6573-6592, 2015.

[45] M. Salucci, G. Oliveri, A. Randazzo, M. Pastorino, and A. Massa, "Electromagnetic subsurface prospecting by a fully nonlinear multifocusing inexact Newton method", J. of the Opt. Soc. of America A, vol. 31, no. 12, pp. 2618-2629, 2014.

[46] L. Poli, G. Oliveri, P. P. Ding, T. Moriyama, and A. Massa, "Multifrequency Bayesian compressive sensing methods for microwave imaging", J. of the Opt. Soc. of America A, vol. 31, no. 11, pp. 2415-2428, 2014. 
[47] A. Massa, P. Rocca, and G. Oliveri, "Compressive sensing in electromagnetics - a review", IEEE Antenn. and Propag.n Mag., vol. 57, no. 1, pp. 224-238, 2015.

[48] M. Ambrosanio and V. Pascazio, "A compressive-sensing-based approach for the detection and characterization of buried objects", IEEE J. of Selec.Topics in Appl. Earth Observ. and Remote Sensing, vol. 8, no. 7, pp. 3386-3395, 2015.

[49] C. Estatico, A. Fedeli, M. Pastorino, and A. Randazzo, "Buried object detection by means of a Lp Banach-space inversion procedure", Radio Science, vol. 50, no. 1, pp. 41-51, 2015.

[50] C. Estatico, A. Fedeli, M. Pastorino, and A. Randazzo, "A multifrequency inexact-Newton method in Lp Banach spaces for buried objects detection", IEEE Trans. on Antenn. and Propag., vol. 63, no. 9, pp. 4198-4204, 2015.

[51] I. Catapano, A. Affinito, G. Gennarelli, F. d. Maio, A. Loperte, and F. Soldovieri, "Full three-dimensional imaging via ground penetrating radar: assessment in controlled conditions and on field for archaeological prospecting", Appl. Phys. A, vol. 115, no. 4, pp. 1415-1422, 2013.

[52] I. Catapano, L. Crocco, Y. Krellmann, G. Triltzsch, and F. Soldovieri, "Tomographic airborne ground penetrating radar imaging: Achievable spatial resolution and on-field assessment", ISPRS J. of Photogramm. and Remote Sensing, vol. 92, pp. 69-78, 2014.

[53] R. Solimene, G. Leone, and A. Dell'Aversano, "MUSIC algorithms for rebar detection", J. of Geophys. and Engin., vol. 10, no. 6, p. 064006, 2013.

[54] I. Catapano, L. Crocco, and T. Isernia, "Improved sampling methods for shape reconstruction of 3-D buried targets", IEEE Trans. on Geosci. and Remote Sensing, vol. 46, no. 10, pp. 3265-3273, 2008.

[55] L. Di Donato, M. T. Bevacqua, L. Crocco, and T. Isernia, "Inverse scattering via virtual experiments and contrast source regularization", IEEE Trans. on Antenn. and Propag., vol. 63, no. 4, pp. 1669-1677, 2015.

[56] L. Di Donato and L. Crocco, "Model-based quantitative crossborehole GPR imaging via virtual experiments", IEEE Trans. on Geosc. and Remote Sensing, vol. 53, no. 8, pp. 4178-4185, 2015.

[57] A. Sahin and E. L. Miller, "Object detection using high resolution near-field array processing", IEEE Trans. on Geosci. and Remote Sensing, vol. 39, no. 1, pp. 136-141, 2001.

[58] S. Meschino, L. Pajewski, M. Pastorino, A. Randazzo, and G. Schettini, "Detection of subsurface metallic utilities by means of a SAP technique: Comparing MUSIC- and SVM-based approaches", J. of Appl. Geophys., vol. 97, pp. 60-68, 2013.

[59] S. Meschino, L. Pajewski, and G. Schettini, "A SAP-DoA method for the localization of two buried objects", Int. J. of Antenn. and Propag., vol. 2013, Article ID e702176, pp. 1-10, 2013 (doi: 10.1155/2013/702176).

[60] E. Bermani, A. Boni, S. Caorsi, and A. Massa, "An innovative realtime technique for buried object detection", IEEE Trans. on Geosci. and Remote Sensing, vol. 41, no. 4, pp. 927-931, 2003.

[61] R. Solimene, A. Cuccaro, A. Dell'Aversano, I. Catapano, and F. Soldovieri, "Background removal methods in GPR prospecting", in Proc. Eur. Radar Conf. EuRAD 2013, Nuremberg, Germany, 2013, pp. $85-88$.

[62] S. Antonijevic and D. Poljak, "A novel time-domain reflection coefficient function: TM case", IEEE Trans. on Electromag. Compatibil., vol. 55, no. 6, pp. 1147-1153, 2013.

[63] C. Le Bastard, Y. Wang, V. Baltazart, and X. Dérobert, “Time delay and permittivity estimation by ground-penetrating radar with support vector regression", IEEE Geosci. and Remote Sensing Lett., vol. 11, no. 4, pp. 873-877, 2014.

[64] M. Sun, N. Pinel, C. L. Bastard, V. Baltazart, A. Ihamouten, and Y. Wang, "Time delay and surface roughness estimation by subspace algorithms for pavement survey by radar", in 7th Int. Worksh. on Adv. Ground Penetrating Radar IWAGPR 2013, Nantes, France, 2013, pp. 1-6.
[65] A. Di Matteo, E. Pettinelli, and E. Slob, "Early-time GPR signal attributes to estimate soil dielectric permittivity: A theoretical study", IEEE Trans. on Geosci. and Remote Sensing, vol. 51, no. 3, pp. $1643-1654,2013$.

[66] N. Gueting, A. Klotzsche, J. van der Kruk, J. Vanderborght, $\mathrm{H}$. Vereecken, and A. Englert, "Imaging and characterization of facies heterogeneity in an alluvial aquifer using GPR full-waveform inversion and cone penetration tests", J. of Hydrology, vol. 524, pp. 680-695, 2015.

[67] A. R. Mangel, S. M. J. Moysey, and J. van der Kruk, "Resolving precipitation induced water content profiles by inversion of dispersive GPR data: A numerical study", J. of Hydrology, vol. 525, pp. 496-505, 2015.

[68] S. Lambot, E. C. Slob, I. van den Bosch, B. Stockbroeckx, and M. Vanclooster, "Modeling of Ground-Penetrating Radar for accurate characterization of subsurface electric properties", IEEE Trans. on Geosci. and Remote Sensing, vol. 42, no. 11, pp. 2555-2568, 2004.

[69] F. André and S. Lambot, "Intrinsic modeling of near-field electromagnetic induction antennas for layered medium characterization. IEEE Trans. on Geosci. and Remote Sensing, vol. 52, no. 11, pp. 7457-7469, 2014.

[70] F. Soldovieri, O. Lopera, and S. Lambot, "Combination of advanced inversion techniques for an accurate target localization via GPR for demining applications", IEEE Trans. on Geosci. and Remote Sensing, vol. 49, no. 1, pp. 451-461, 2011.

[71] A. P. Tran, F. André, and S. Lambot, "Validation of near-field Ground-Penetrating Radar modeling using full-wave inversion for soil moisture estimation", IEEE Trans. on Geosci. and Remote Sensing, vol. 52, no. 9, pp. 5483-5497, 2014.

[72] A. De Coster, A. P. Tran, and S. Lambot, "Fundamental analyses on layered media reconstruction using GPR and full-wave inversion in near-field conditions", IEEE Trans. on Geosci. and Remote Sensing, vol. 54, no. 9, pp. 5143-5158, 2016.

[73] L. Pajewski, "The open database of experimental and synthetic GPR radargrams", COST Action TU1208, 2016 [Online]. Available: http://gpradar.eu/onewebmedia/TU1208\%20TS\%20Split\%20\%20Pajewski\%20-\%20Database\%20of\%20radargrams.pdf

[74] "GPR imaging challenge", 9th Int. Worksh. on Advanced Ground Penetrating Radar IWAGPR 2017, Edinburgh, Scotland, 2017 [Online]. Available: https://github.com/gprMax/iwagpr2017-model

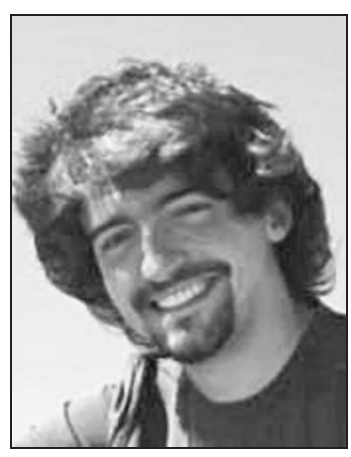

Alessandro Fedeli received the B.Sc. and the M.Sc. in Electronic Engineering from the University of Genoa (Italy) in 2011 and 2013, respectively. In 2017 he received the Ph.D. degree in Science and Technology for Electronic and Telecommunications Engineering from the same University. His research activity is mainly focused on microwave imaging and computational methods for the solution of forward and inverse electromagnetic scattering problems. He is interested in biomedical imaging applications and advanced processing techniques for Ground Penetrating Radar (GPR) systems. He is WG Member of the COST Action TU1208 "Civil Engineering Applications of Ground Penetrating Radar", and Member of the IEEE Antennas and Propagation Society. 
E-mail: alessandro.fedeli@edu.unige.it

Department of Electrical, Electronic, Telecommunications Engineering, and Naval Architecture

University of Genoa

Via Opera Pia, 11A

I-16145 Genova, Italy

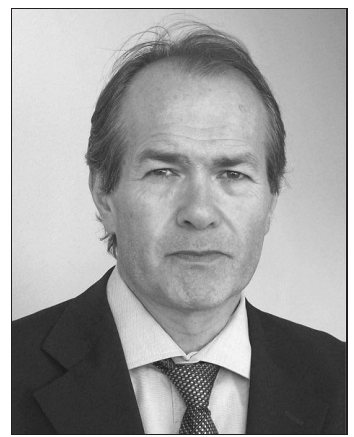

Matteo Pastorino is a Full Professor of electromagnetic fields at the Polytechnic School of the University of Genoa, where he has been the past Director of the Department of Biophysical and Electronic Engineering (DIBE, 2008-2011) and the past Director of the Department of Electrical, Electronic, Telecommunications Engineering and Naval Architecture (DITEN, 2011-2013). At present, he is a Fellow of the IEEE, the Chair of the National URSI Commission B (Fields and Waves), the responsible for the local section of the National Society of Electromagnetics (SIEM), and the chair of the local section of the Interuniversity Center for the Interaction between Electromagnetic Fields and Biosystems (ICEmB). He has coauthored about 450 papers in international journals and conference proceedings in the field of applied electromagnetics. Prof. Pastorino is an Associate Editor of the IEEE Antennas and Propagation Magazine and of the IEEE Transactions on Instrumentation and Measurement.
E-mail: matteo.pastorino@unige.it

Department of Electrical, Electronic, Telecommunications Engineering, and Naval Architecture

University of Genoa

Via Opera Pia, 11A

I-16145 Genova, Italy

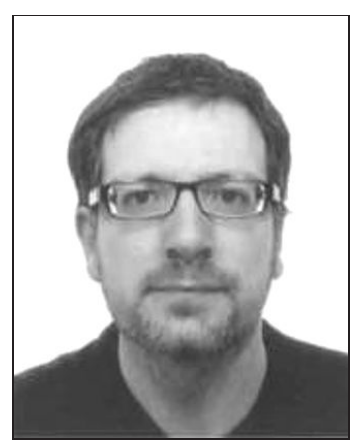

Andrea Randazzo received the laurea degree in Telecommunication Engineering from the University of Genoa, Genoa, Italy, in 2001 and the Ph.D. degree in Information and Communication Technologies from the same university in 2006. Currently, he is an Associate Professor at the Department of Electrical, Electronic, Telecommunications Engineering, and Naval Architecture of the University of Genoa. His primary research interests are in the field of electromagnetic scattering (both direct and inverse), smart antennas, and numerical methods for microwave nondestructive evaluations and imaging.

E-mail: andrea.randazzo@unige.it

Department of Electrical, Electronic, Telecommunications Engineering, and Naval Architecture

University of Genoa

Via Opera Pia, 11A

I-16145 Genova, Italy 